\title{
The prediction of repeated sprint and speed endurance performance by parameters of critical velocity models in soccer
}

Erdal Ar1 ${ }^{1 \mathrm{ABCD}}$, Gökhan Deliceoglu ${ }^{2 \mathrm{ABCD}}$

${ }^{1}$ Ordu University, Turkey

${ }^{2}$ Gazi University, Turkey

Authors' Contribution: A - Study design; B - Data collection; C - Statistical analysis; D - Manuscript Preparation; E - Funds Collection

\begin{tabular}{|c|c|}
\hline \\
\hline Purpose: & $\begin{array}{l}\text { The prediction of running anaerobic sprint test and } 800 \mathrm{~m} \text { performance by parameters of critical velocity } \\
\text { was examined in this study. }\end{array}$ \\
\hline Material: & $\begin{array}{l}\text { The participants of study were consisted of thirteen amateur soccer players ( } \mathrm{n}=13, \text { age }=22.69 \pm 5.29 \text { years, } \\
\text { weight }=72.46 \pm 6.32 \mathrm{~kg} \text {, height }=176.92 \pm 6.73 \mathrm{~cm} \text { ). The } 800 \text { and } 2400 \mathrm{~m} \text { running tests were performed for } \\
\text { determination of critical velocity and anaerobic distance capacity. The critical velocity and anaerobic } \\
\text { distance capacity were determined by three mathematical models (linear total distance, linear velocity, } \\
\text { non-linear two parameter model). The repeated sprint and sprint endurance ability was determined by } \\
\text { running anaerobic sprint test and } 800 \mathrm{~m} \text { running test. The simple and multiple linear regression analysis } \\
\text { was used for prediction of dependent variables (running anaerobic sprint test and } 800 \mathrm{~m} \text { running } \\
\text { performance) by independent variables (critical velocity and anaerobic distance capacity) of study. The } \\
\text { correlation between variables was determined by Pearson correlation coefficient. }\end{array}$ \\
\hline Results: & $\begin{array}{l}\text { It was found that anaerobic distance capacity was a significant predictor of running anaerobic sprint test } \\
\text { and } 800 \mathrm{~m} \text { running performance }(\mathrm{p}<0.05) \text {. However, it was determined that critical velocity predicted } \\
\text { significantly only time parameters of running anaerobic sprint test and } 800 \mathrm{~m} \text { test }(\mathrm{p}<0.05) \text {. Also, the } \\
\text { parameters of } 800 \mathrm{~m} \text { test (except for average velocity) were significantly predicted by running anaerobic } \\
\text { sprint test parameters }(\mathrm{p}<0.05) \text {. }\end{array}$ \\
\hline Conclusions: & $\begin{array}{l}\text { It may be concluded that anaerobic distance capacity is an indicator of repeated sprint and speed } \\
\text { endurance ability in soccer and may be used in improvement of sprint endurance performance. }\end{array}$ \\
\hline ywords: & \\
\hline
\end{tabular}

\section{Introduction}

The oxygen uptake of muscles may be accepted as important determinant of aerobic exercise performance and critical velocity $(\mathrm{CV})$ or critical power parameter is closely related to it. It is indicated that the critical power is an important indicator of oxygen uptake ability during exercise [1]. The $\mathrm{CV}$ was originated from critical power concept. The critical power concept was firstly defined by Monod and Scherrer [2]. The critical power tests were consisted of a series of exhausting exercises on small muscle groups with different exercise intensities [2]. The critical power (slope of linear regression graph) and anaerobic work capacity (y-intercept of linear regression graph) parameters were yielded by the linear relationship between work (parameter determined by multiplied of power and time) and exhaustion time of test [4-6]. Then, test procedure was performed on cycle ergometer with different power values [3]. The CV and anaerobic distance capacity (ADC) were determined by a series tests performed on treadmill [7,8]. The CV and ADC parameters corresponded to critical power and anaerobic work capacity parameters in critical power test $[9,10]$. The $\mathrm{CV}$ is maximum running velocity sustained without fatigue and $\mathrm{ADC}$ is distance covered with anaerobic energy sources in muscles [9-11]. The aerobic fitness level of

(c) Erdal Arı, Gökhan Deliceoglu, 2021

doi:10.15561/26649837.2021.0208 athletes may be evaluated with $\mathrm{CV}$ and ADC parameters. The CV tests have various advantages at determination of aerobic fitness. The procedure of $\mathrm{CV}$ tests is simple and easy for aerobic performance measurement of athletes. The CV parameter was determined with distance, velocity and time parameters of two or more runnings. Also, the $\mathrm{CV}$ test protocol may be performed on treadmill or field.

The aerobic exercises such as walking, runnings with low intensity are frequently performed during soccer game frequently. On the other hand, it was indicated that the explosive power activities, explosive runnings, jumps, repeated sprints are important decisive of match performance [12-15]. The repeated sprint ability may relate to performance at last parts of soccer match observed fatigue. The anaerobic activities such as sprint may have high importance on critical moments of soccer match [16]. The anaerobic activities such as repeated sprints may be related to various performance parameters as CV and ADC. If the probable effects on anaerobic activities (repeated sprint and $800 \mathrm{~m}$ ) of CV and ADC parameters is determined, the anaerobic activities of soccer players may be organized in the light of CV and ADC parameters. Many studies are available in literature about anaerobic activities such as repeated sprint and $800 \mathrm{~m}$ running. However, it has been seen that few studies in literature have focused on relationship between $\mathrm{CV}$ test parameters and anaerobic activities such as repeated sprint and 800 
$\mathrm{m}$. The aim of this study was to predict repeated sprint and $800 \mathrm{~m}$ performances by $\mathrm{CV}$ and ADC parameters.

\section{Material and Methods}

\section{Participants}

The thirteen amateur soccer players $(n=13$, age $=22.69 \pm 5.29$ years, weight $=72.46 \pm 6.32 \quad \mathrm{~kg}$, height $=176.92 \pm 6.73 \mathrm{~cm}$ ) participated in the study. The participants consisted of players playing in a soccer team competing regional amateur league of Turkey and performing soccer trainings 1.5 hours for five days of a week regularly. The participants were informed about study and they signed informed voluntary consent form. The study was performed according to principles of Helsinki Declaration.

Research Design

Data Collection

$800 \mathrm{~m}$ and $2400 \mathrm{~m}$ Running Tests

The 800 and $2400 \mathrm{~m}$ running tests were performed in order to determine CV and ADC [17]. The tests were performed on synthetic grass soccer pitch at same hour of day to eliminate effects of circadian rhythm. The high intensity exercises were not performed within 24 hours before the tests. The measurements were performed at preseason preparation term. The distance of tests was marked with training cones. The $800 \mathrm{~m}$ test was firstly performed firstly. The players performed ten minutes warm-up and stretching exercises before the test. Each player entered the test individually. The players tried to run $800 \mathrm{~m}$ distance in shortest time with maximum effort (with 100\% exercise intensity). The verbal encouragement was given to players by researchers during test. The test was finished when $800 \mathrm{~m}$ distance was covered by players. The test time was measured by wireless photocell system (Witty, Microgate, Bolzano, Italy). The test time was recorded in second unit with 0.01 precision. After three days from $800 \mathrm{~m}$ test, $2400 \mathrm{~m}$ test was performed by players. The procedure of $2400 \mathrm{~m}$ test was similar to the $800 \mathrm{~m}$ test. The test time of players was measured similarly. After the tests, the players performed warm-down exercises.

\section{Running Anaerobic Sprint Test (RAST)}

RAST was a test used for determination of repeated sprint ability $[18,19]$. The RAST was consisted of six 35 $\mathrm{m}$ sprints performed with 10 seconds rest interval. The test was carried out on synthetic grass soccer pitch at same hour of day after three days from $2400 \mathrm{~m}$ running test. The $35 \mathrm{~m}$ test track was set on soccer pitch. The gates of wireless photocell system (Witty, Microgate, Bolzano, Italy) were placed at start and finish point of $35 \mathrm{~m}$ test track. The players performed warm-up and stretching exercises before test. The players performed six $35 \mathrm{~m}$ sprints with 10 seconds recovery interval between each sprint. The verbal motivation was given to players during test. The time of six sprints was recorded in second unit with 0.01 precision. The test parameters were determined as follow:

- $\quad$ Power $($ watt $)=$ Weight $(\mathrm{kg}) \times$ Distance $(\mathrm{m})^{2} \div$ Time ${ }^{3}(\mathrm{sec})[20]$,

- Minimum power (watt): The lowest power value of six sprints,

- $\quad$ Peak power (watt): The highest power value of six sprints,

- $\quad$ Average power (watt): The mean power value of six sprints,

- $\quad$ Fatigue index $($ watt $/ \mathrm{sec})=($ Maximum power $($ watt $)-$ Minimum power (watt) $) \div$ Total time of 6 sprints (sec) [18],

- Average test time (sec): Mean of six sprint times,

- Total test time (sec): Sum of six sprints times,

- Velocity $(\mathrm{km} / \mathrm{h})=$ Sprint distance $(\mathrm{km}) \div$ Sprint time (h),

- Average velocity $(\mathrm{km} / \mathrm{h})$ : Mean velocity of six sprints,

- Maximum velocity $(\mathrm{km} / \mathrm{h})$ : The highest velocity of six sprints.

800 m Running Test

The $800 \mathrm{~m}$ test was carried out in order to determine relationship between $800 \mathrm{~m}$ performance and RAST, CV and ADC parameters. After three days from RAST, $800 \mathrm{~m}$ test was performed at same hour of day on synthetic grass soccer pitch. Test track was prepared on soccer pitch. After warm-up and stretching exercises, players started the test. Each player performed the test individually. The players ran with maximum effort and covered 800 $\mathrm{m}$ distance at shortest time. Test time was determined by wireless photocell system (Witty, Microgate, Bolzano, Italy). The $800 \mathrm{~m}$ test time was recorded in sec unit with 0.01 precision.

Determination of Critical Velocity $(\mathrm{CV})$ and Anaerobic Distance Capacity (ADC)

The three mathematical models were used for determination of $\mathrm{CV}$ and $\mathrm{ADC}$. These mathematical models consisted of linear and non-linear regression models. The time (t), distance (D) and velocity (V) at 800 and $2400 \mathrm{~m}$ tests were used in three mathematical models. The first mathematical model was linear total distance (Lin-TD) model. The Lin-TD model was derived from linear regression analysis between $\mathrm{D}$ and $\mathrm{t}$ parameters of 800 and $2400 \mathrm{~m}$ tests for each participant [20-27]:

$$
\mathrm{D}=\mathrm{ADC}+\mathrm{CV} \times \mathrm{t}
$$

In Lin-TD model, the regression slope was $\mathrm{CV}$ and $y$-intercept of distance-time relationship (y-intercept of regression line) was ADC.

The second mathematical model was linear velocity (Lin-V) model. The Lin-V model was consisted of regression analysis between $\mathrm{V}$ and inverse of time $(1 / \mathrm{t})$ of 800 and $2400 \mathrm{~m}$ tests and the $1 / \mathrm{t}$ value were used in model to be converted to linear of hyperbolic relationship between $\mathrm{V}$ and $\mathrm{t}$ [23-25, 27-31]:

$$
\mathrm{V}=\operatorname{ADC} \times(1 / \mathrm{t})+\mathrm{CV}
$$

In Lin-V model, the regression slope was ADC and $y$-intercept of $\mathrm{V}-1 / \mathrm{t}$ relationship (y-intercept of regression line) was $\mathrm{CV}$.

The third mathematical model was known as nonlinear model with 2-parameter (Non-2). The equation 
of Lin- $\mathrm{V}$ model was solved for $\mathrm{t}$ parameter and the hyperbolic relationship between $\mathrm{V}$ and $\mathrm{t}$ was indicated by Non-2 model [27-30, 32-34]:

$$
\mathrm{t}=\mathrm{ADC} /(\mathrm{V}-\mathrm{CV})
$$

\section{Statistical Analysis}

The descriptive statistics of study were presented as mean \pm standard deviation and range (minimummaximum) values (table 1 ). The $\mathrm{CV}$ and $\mathrm{ADC}$ parameters of each player were determined by linear and nonlinear regression analysis. The Shapiro Wilk test was utilised to examine normality of data. The simple scatter graphs were examined for determination of linearity between dependent and independent variables at regression models. The multicollinearity between independent variables of regression models was examined by VIF (variance inflation factor). The regression models were designed in accordance with ideal VIF. The simple and multiple linear regression analysis was used for prediction of dependent variables (RAST and $800 \mathrm{~m}$ test parameters) by independent variables of study (CV and ADC). All data were analysed in the SPSS package program (IBM SPSS 22.0. Armonk, NY: IBM Corp.). The significance level at statistical analysis was performed as $\mathrm{p}<0.05$.

\section{Results}

Table 1. The Descriptive Statistics of Test Parameters
There was no significant correlation between $\mathrm{CV}$ values and RAST parameters in Table $2(\mathrm{p}>0.05)$. On the other hand, it was found that the ADC values correlated significantly with RAST parameters $(\mathrm{p}<0.05)$.

According to analysis results in Table 3 , it was seen that $\mathrm{CV}$ and $\mathrm{ADC}$ parameters correlated significantly with time and maximum velocity at $800 \mathrm{~m}$ test $(\mathrm{p}<0.05)$. It was found no significant correlation between average velocity at $800 \mathrm{~m}$ test and $\mathrm{CV}, \mathrm{ADC}$ parameters $(\mathrm{p}>0.05)$.

According to results of correlation analysis between RAST and $800 \mathrm{~m}$ test parameters in Table 4, there was a significant correlation between RAST parameters and time and maximum velocity at $800 \mathrm{~m}$ test $(\mathrm{p}<0.05)$. It was found that there was no significant correlation between average velocity at $800 \mathrm{~m}$ test and RAST parameters $(\mathrm{p}>0.05)$.

In Table 5, the analysis result indicated prediction of the RAST parameters by $\mathrm{CV}$ and ADC parameters. The both $\mathrm{CV}$ and $\mathrm{ADC}$ predicted significantly time parameters of RAST $\left(\mathrm{t}_{\text {verage }}\right.$ and $\left.\mathrm{t}_{\text {total }}\right)(\mathrm{p}<0.05)$. The velocity and power parameters of RAST $\left(\mathrm{V}_{\text {average }}, \mathrm{V}_{\text {max }}\right.$, minimum, maximum and mean power) were significantly predicted by ADC merely $(p<0.05)$. Also, it was found that the ADC of Lin-TD and Non-2 model was a significant predictor of fatigue index value of RAST $(\mathrm{p}<0.05)$.

According to results in Table 6, the parameters of time and maximum velocity at $800 \mathrm{~m}$ test were significantly

\begin{tabular}{|c|c|c|c|c|}
\hline Tests & & Parameters & Mean \pm SD & Range \\
\hline \multirow{6}{*}{ CV Test ( $n=13)$} & \multirow{2}{*}{ Lin-TD Model } & $\mathrm{CV}(\mathrm{km} / \mathrm{h})$ & $12.93 \pm 0.61$ & $11.95-14.00$ \\
\hline & & $\mathrm{ADC}(\mathrm{km})$ & $0.22 \pm 0.03$ & $0.15-0.28$ \\
\hline & \multirow{2}{*}{ Lin-V Model } & $\mathrm{CV}(\mathrm{km} / \mathrm{h})$ & $12.96 \pm 0.61$ & $11.93-14.01$ \\
\hline & & $\mathrm{ADC}(\mathrm{km})$ & $0.22 \pm 0.03$ & $0.16-0.28$ \\
\hline & \multirow{2}{*}{ Non-2 Model } & $\mathrm{CV}(\mathrm{km} / \mathrm{h})$ & $12.96 \pm 0.62$ & $11.98-14.01$ \\
\hline & & $\mathrm{ADC}(\mathrm{km})$ & $0.22 \pm 0.03$ & $0.15-0.28$ \\
\hline \multirow{11}{*}{ RAST Test $(n=13)$} & & $\mathrm{t}_{\text {(average) }}(\mathrm{sec})$ & $5.23 \pm 0.27$ & $4.87-5.81$ \\
\hline & & $\mathrm{t}_{\text {(total) }}(\mathrm{sec})$ & $31.40 \pm 1.64$ & $29.20-34.85$ \\
\hline & & $\mathrm{V}_{\text {(average) }}(\mathrm{km} / \mathrm{h})$ & $24.20 \pm 1.22$ & $21.75-25.95$ \\
\hline & & $\mathrm{V}_{(\max )}(\mathrm{km} / \mathrm{h})$ & $26.08 \pm 1.35$ & $23.38-27.88$ \\
\hline & & Min. power (relative) $_{(\text {watt} / \mathrm{kg})}$ & $7.04 \pm 1.06$ & $5.27-8.92$ \\
\hline & & Min. power (watt) & $509.89 \pm 92.50$ & $393.57-713.31$ \\
\hline & & Max. power $r_{\text {(relative) }}($ watt/kg) & $10.94 \pm 1.66$ & $7.82-13.27$ \\
\hline & & Max. power (watt) & $791.76 \pm 135.62$ & $589.85-1061.23$ \\
\hline & & Mean power ${ }_{\text {(relative) }}($ watt $/ \mathrm{kg})$ & $8.82 \pm 1.29$ & $6.36-10.78$ \\
\hline & & Mean power (watt) & $638.38 \pm 108.38$ & $479.26-862.42$ \\
\hline & & Fatigue index (watt/sec) & $9.05 \pm 2.42$ & $5.53-14.12$ \\
\hline \multirow{3}{*}{\multicolumn{2}{|c|}{$800 m$ running test $(n=13)$}} & $\mathrm{t}(\mathrm{sec})$ & $155.00 \pm 9.35$ & $145.00-179.00$ \\
\hline & & $\mathrm{V}_{\text {(average) }}(\mathrm{km} / \mathrm{h})$ & $18.30 \pm 1.43$ & $15.00-20.00$ \\
\hline & & $V_{\max }(\mathrm{km} / \mathrm{h})$ & $24.50 \pm 2.33$ & $19.60-27.70$ \\
\hline
\end{tabular}

Note. Lin-TD: Linear Total Distance Model, Lin-V:Linear Velocity Model, Non-2: Nonlinear 2-parameter Model, CV: Critical velocity, ADC: Anaerobic Distance Capacity, RAST: Repeated Anaerobic Sprint Test, $\mathrm{t}$ : test time, $\mathrm{t}_{\text {(average) }}=$ average test time, $\mathrm{t}_{\text {(total) }}=$ total test time, $\mathrm{V}$ : velocity, $\mathrm{V}_{\text {(average) }}=$ average velocity, $\mathrm{V}_{\text {(max) }}=$ maximum velocity, min. power $_{\text {(relative) }}=$ relative minimum power of six $35 \mathrm{~m}$. runnings, min.power=salt minimum power of six $35 \mathrm{~m}$. runnings, max. power ${ }_{\text {(relative) }}=$ relative maximum power of six $35 \mathrm{~m}$. runnings, max. power=salt maximum power of six $35 \mathrm{~m}$. runnings, mean power ${ }_{\text {(relative) }}$ relative mean power of six $35 \mathrm{~m}$. runnings, mean power=salt mean power of six $35 \mathrm{~m}$. runnings, fatigue index=fatigue index of six $35 \mathrm{~m}$. runnings. 
Table 2. Pearson Correlation Analysis Results Between CV, ADC and RAST Parameters

\begin{tabular}{|c|c|c|c|c|c|c|c|c|c|c|}
\hline \multirow[b]{2}{*}{$\begin{array}{l}\bar{d} \\
\bar{\Sigma}\end{array}$} & \multirow[b]{2}{*}{ 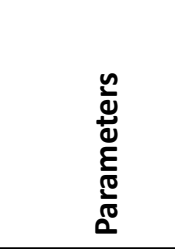 } & \multirow[b]{2}{*}{$\begin{array}{l}\frac{1}{0} \\
\frac{0}{\pi} \\
\frac{0}{0} \\
0 \\
0\end{array}$} & \multicolumn{8}{|l|}{ RAST } \\
\hline & & & $\begin{array}{l}t_{\text {(average) }} \\
\text { (sec) }\end{array}$ & $\begin{array}{l}\mathbf{t}_{\text {(total) }} \\
\text { (sec) }\end{array}$ & $\begin{array}{l}V_{\text {(average) }} \\
(\mathrm{km} / \mathrm{h})\end{array}$ & $\begin{array}{l}V_{(\max )} \\
(\mathrm{km} / \mathrm{h})\end{array}$ & $\begin{array}{l}\text { Min. } \\
\text { power }_{\text {(relative) }} \\
\text { (watt) }\end{array}$ & $\begin{array}{l}\text { Max. } \\
\text { power }_{\text {(relative) }} \\
\text { (watt) }\end{array}$ & $\begin{array}{l}\text { Mean } \\
\text { power }_{\text {(relative) }} \\
\text { (watt) }\end{array}$ & $\begin{array}{l}\text { Fatigue } \\
\text { index } \\
\text { (watt/ } \\
\text { sec) }\end{array}$ \\
\hline \multirow{4}{*}{ Lin-TD } & \multirow{2}{*}{$\mathrm{CV}(\mathrm{km} / \mathrm{h})$} & $r$ & -0.500 & -0.501 & 0.480 & 0.411 & 0.438 & 0.384 & 0.457 & 0.135 \\
\hline & & $p$ & 0.082 & 0.081 & 0.097 & 0.163 & 0.135 & 0.195 & 0.116 & 0.661 \\
\hline & \multirow{2}{*}{$\mathrm{ADC}(\mathrm{km})$} & $r$ & -0.635 & -0.637 & 0.629 & 0.671 & 0.594 & 0.666 & 0.621 & 0.593 \\
\hline & & $\mathrm{p}$ & $0.020 *$ & $0.019^{*}$ & $0.021^{*}$ & $0.012^{*}$ & $0.032 *$ & $0.013^{*}$ & $0.024 *$ & $0.033^{*}$ \\
\hline \multirow{4}{*}{ Lin-V } & \multirow{2}{*}{$\mathrm{CV}(\mathrm{km} / \mathrm{h})$} & $r$ & -0.504 & -0.505 & 0.485 & 0.433 & 0.424 & 0.407 & 0.463 & 0.199 \\
\hline & & $\mathrm{p}$ & 0.079 & 0.078 & 0.093 & 0.140 & 0.149 & 0.167 & 0.111 & 0.515 \\
\hline & \multirow{2}{*}{$\mathrm{ADC}(\mathrm{km})$} & $r$ & -0.696 & -0.697 & 0.693 & 0.679 & 0.721 & 0.675 & 0.688 & 0.506 \\
\hline & & $\mathrm{p}$ & $0.008^{*}$ & $0.008^{*}$ & $0.009^{*}$ & $0.011^{*}$ & $0.005^{*}$ & $0.011 *$ & $0.009 *$ & 0.078 \\
\hline \multirow{4}{*}{ Non-2 } & \multirow{2}{*}{$\mathrm{CV}(\mathrm{km} / \mathrm{h})$} & $r$ & -0.504 & -0.505 & 0.485 & 0.414 & 0.446 & 0.388 & 0.462 & 0.137 \\
\hline & & $\mathrm{p}$ & 0.079 & 0.078 & 0.093 & 0.160 & 0.127 & 0.191 & 0.112 & 0.656 \\
\hline & \multirow{2}{*}{$\mathrm{ADC}(\mathrm{km})$} & $r$ & -0.633 & -0.635 & 0.626 & 0.668 & 0.596 & 0.662 & 0.618 & 0.596 \\
\hline & & $\mathrm{p}$ & $0.020 *$ & $0.020^{*}$ & $0.022^{*}$ & $0.013^{*}$ & $0.032^{*}$ & $0.014^{*}$ & $0.024^{*}$ & $0.031^{*}$ \\
\hline
\end{tabular}

Note. ${ }^{*} p<0.05$

Table 3. Pearson Correlation Analysis Results Between CV, ADC and $800 \mathrm{~m}$ Test Parameters

\begin{tabular}{|c|c|c|c|c|c|}
\hline \multirow[b]{2}{*}{ Model } & \multirow[b]{2}{*}{ Parameters } & \multirow[b]{2}{*}{ Correlation } & \multicolumn{3}{|c|}{$800 \mathrm{~m}$ test } \\
\hline & & & $\begin{array}{l}t \\
(\mathrm{sec})\end{array}$ & $\begin{array}{l}V_{\text {(average) }} \\
(\mathrm{km} / \mathrm{h})\end{array}$ & $\begin{array}{l}V_{\max )} \\
(\mathrm{km} / \mathrm{h})\end{array}$ \\
\hline \multirow{4}{*}{ Lin-TD } & \multirow{2}{*}{$\mathrm{CV}(\mathrm{km} / \mathrm{h})$} & $r$ & -0.452 & -0.064 & 0.555 \\
\hline & & $p$ & 0.121 & 0.837 & $0.049 *$ \\
\hline & \multirow{2}{*}{$\mathrm{ADC}(\mathrm{km})$} & $r$ & -0.770 & 0.510 & 0.681 \\
\hline & & $\mathrm{p}$ & $0.002^{*}$ & 0.075 & $0.010 *$ \\
\hline \multirow{4}{*}{ Lin-V } & \multirow{2}{*}{$\mathrm{CV}(\mathrm{km} / \mathrm{h})$} & $r$ & -0.475 & -0.071 & 0.565 \\
\hline & & $p$ & 0.101 & 0.817 & $0.044^{*}$ \\
\hline & \multirow{2}{*}{$\mathrm{ADC}(\mathrm{km})$} & $r$ & -0.782 & 0.499 & 0.726 \\
\hline & & $\mathrm{p}$ & $0.002^{*}$ & 0.082 & $0.005^{*}$ \\
\hline \multirow{4}{*}{ Non-2 } & \multirow{2}{*}{$\mathrm{CV}(\mathrm{km} / \mathrm{h})$} & $r$ & -0.451 & -0.074 & 0.560 \\
\hline & & $\mathrm{p}$ & 0.122 & 0.810 & $0.047^{*}$ \\
\hline & \multirow{2}{*}{$\mathrm{ADC}(\mathrm{km})$} & $r$ & -0.762 & 0.493 & 0.686 \\
\hline & & $\mathrm{p}$ & $0.002^{*}$ & 0.087 & $0.010^{*}$ \\
\hline
\end{tabular}

Note. ${ }^{*} p<0.05$

predicted by both $\mathrm{CV}$ and $\mathrm{ADC}(\mathrm{p}<0.05)$. Also, it was seen that $\mathrm{CV}$ and $\mathrm{ADC}$ were not significant predictors of average velocity at $800 \mathrm{~m}$ test $(\mathrm{p}>0.05)$.

The results in Table 7 indicated that the RAST parameters were significant predictors of time and maximum velocity at $800 \mathrm{~m}$ test $(\mathrm{p}<0.05)$. The average velocity at $800 \mathrm{~m}$ test was not significantly predicted by RAST parameters $(p>0.05)$. On the other hand, it was found that the fatigue index parameter of RAST predicted significantly time of $800 \mathrm{~m}$ test merely $(\mathrm{p}<0.05)$.

\section{Discussion}

The CV and ADC parameters are products of linear relationship between distance and time of exercise. Also, these parameters are determined from linear relationship between velocity and time ${ }^{-1}$ or other nonlinear mathematical models. The $\mathrm{CV}$ and $\mathrm{ADC}$ were frequently investigated in various studies. It was found that $\mathrm{CV}$ correlated with maximal aerobic velocity and maximum oxygen uptake $[35,36]$. The significant and high correlation $(r=0.80-0.93$ range, $\mathrm{p}<0.01)$ between $\mathrm{CV}$ of five mathematical models and one hour running performance indicated relationship between $\mathrm{CV}$ and 
Table 4. Pearson Correlation Analysis Results Between RAST and 800 m Test Parameters

\begin{tabular}{|c|c|c|c|c|}
\hline \multirow{2}{*}{ RAST Parameters } & \multirow{2}{*}{ Correlation } & \multicolumn{3}{|c|}{$800 \mathrm{~m}$ test } \\
\hline & & $t(\sec )$ & $\mathrm{V}_{\text {(average) }}(\mathrm{km} / \mathrm{h})$ & $V_{(\max )}(\mathrm{km} / \mathrm{h})$ \\
\hline \multirow{2}{*}{$\mathrm{t}_{\text {(average) }}(\mathrm{sec})$} & $r$ & 0.806 & -0.341 & -0.746 \\
\hline & $p$ & $0.001^{*}$ & 0.254 & $0.003 *$ \\
\hline \multirow{2}{*}{$\mathrm{t}_{\text {(total) }}(\mathrm{sec})$} & $r$ & 0.807 & -0.342 & -0.745 \\
\hline & $p$ & $0.001^{*}$ & 0.253 & $0.003^{*}$ \\
\hline \multirow{2}{*}{$\mathrm{V}_{\text {(average) }}(\mathrm{km} / \mathrm{h})$} & $r$ & -0.799 & 0.335 & 0.725 \\
\hline & $p$ & $0.001^{*}$ & 0.263 & $0.005^{*}$ \\
\hline \multirow{2}{*}{$\mathrm{V}_{(\max )}(\mathrm{km} / \mathrm{h})$} & $r$ & -0.815 & 0.438 & 0.698 \\
\hline & $p$ & $0.001^{*}$ & 0.135 & $0.008^{*}$ \\
\hline \multirow{2}{*}{ Min. power ${ }_{\text {(relative) }}$ (watt) } & $r$ & -0.698 & 0.197 & 0.762 \\
\hline & $p$ & $0.008^{*}$ & 0.519 & $0.002^{*}$ \\
\hline \multirow{2}{*}{ Max. power $_{\text {(relative) }}$ (watt) } & $r$ & -0.804 & 0.434 & 0.678 \\
\hline & $p$ & $0.001^{*}$ & 0.139 & $0.011^{*}$ \\
\hline \multirow{2}{*}{ Mean power $_{\text {(relative) }}$ (watt) } & $r$ & -0.790 & 0.330 & 0.705 \\
\hline & $p$ & $0.001^{*}$ & 0.271 & $0.007^{*}$ \\
\hline \multirow{2}{*}{ Fatigue index (watt/sec) } & $r$ & -0.661 & 0.420 & 0.387 \\
\hline & $p$ & $0.014^{*}$ & 0.153 & 0.191 \\
\hline
\end{tabular}

Note. ${ }^{*} p<0.05$

Table 5. The Regression Analysis of Effect on RAST Parameters of CV and ADC as Predictor Variables

\begin{tabular}{|c|c|c|c|c|c|c|c|c|c|c|}
\hline $\begin{array}{l}\text { Dependent } \\
\text { Variable }\end{array}$ & Model & $\begin{array}{l}\text { Predictor } \\
\text { Variables }\end{array}$ & B & $\begin{array}{l}\text { Standard } \\
\text { Error }\end{array}$ & $\beta$ & $\mathbf{t}$ & $\mathbf{p}$ & $\mathbf{R}$ & $\mathbf{R}^{2}$ & $\begin{array}{l}\text { Standard } \\
\text { Error of } \\
\text { Estimate }\end{array}$ \\
\hline \multirow{9}{*}{$\mathrm{t}_{\text {(average) }}(\mathrm{sec})$} & \multirow{3}{*}{ Model-1 } & Constant & 8.895 & 1.146 & & 7.764 & $0.000^{*}$ & \multirow{3}{*}{0.786} & \multirow{3}{*}{0.618} & \multirow{3}{*}{0.184} \\
\hline & & Lin-TD-CV & -0.205 & 0.086 & -0.464 & -2.371 & 0.039* & & & \\
\hline & & Lin-TD-ADC & -4.543 & 1.462 & -0.608 & -3.107 & $0.011^{*}$ & & & \\
\hline & \multirow{3}{*}{ Model-2 } & Constant & 8.904 & 1.062 & & 8.385 & $0.000^{*}$ & \multirow{3}{*}{0.819} & \multirow{3}{*}{0.671} & \multirow{3}{*}{0.171} \\
\hline & & Lin-V-CV & -0.192 & 0.081 & -0.435 & -2.386 & $0.038^{*}$ & & & \\
\hline & & Lin-V-ADC & -5.328 & 1.496 & -0.649 & -3.561 & $0.005^{*}$ & & & \\
\hline & \multirow{3}{*}{ Model-3 } & Constant & 8.918 & 1.145 & & 7.787 & $0.000^{*}$ & \multirow{3}{*}{0.786} & \multirow{3}{*}{0.618} & \multirow{3}{*}{0.184} \\
\hline & & Non-2-CV & -0.205 & 0.086 & -0.467 & -2.384 & $0.038^{*}$ & & & \\
\hline & & Non-2-ADC & -4.611 & 1.494 & -0.604 & -3.086 & $0.012^{*}$ & & & \\
\hline \multirow{9}{*}{$\mathrm{t}_{\text {(total) }}(\mathrm{sec})$} & \multirow{3}{*}{ Model-1 } & Constant & 53.472 & 6.869 & & 7.785 & $0.000^{*}$ & \multirow{3}{*}{0.788} & \multirow{3}{*}{0.621} & \multirow{3}{*}{1.107} \\
\hline & & Lin-TD-CV & -1.235 & 0.518 & -0.465 & -2.385 & $0.038^{*}$ & & & \\
\hline & & Lin-TD-ADC & -27.414 & 8.765 & -0.610 & -3.128 & $0.011^{*}$ & & & \\
\hline & \multirow{3}{*}{ Model-2 } & Constant & 53.527 & 6.360 & & 8.417 & $0.000^{*}$ & \multirow{3}{*}{0.821} & \multirow{3}{*}{0.674} & \multirow{3}{*}{1.027} \\
\hline & & Lin-V-CV & -1.160 & 0.483 & -0.436 & -2.403 & $0.037^{*}$ & & & \\
\hline & & Lin-V-ADC & -32.149 & 8.961 & -0.651 & -3.588 & $0.005^{*}$ & & & \\
\hline & \multirow{3}{*}{ Model-3 } & Constant & 53.607 & 6.867 & & 7.807 & $0.000^{*}$ & \multirow{3}{*}{0.788} & \multirow{3}{*}{0.621} & \\
\hline & & Non-2-CV & -1.237 & 0.516 & -0.468 & -2.397 & $0.037^{*}$ & & & 1.108 \\
\hline & & Non-2-ADC & -27.818 & 8.961 & -0.606 & -3.104 & $0.011^{*}$ & & & \\
\hline & & Constant & 8.308 & 5.315 & & 1.563 & 0.149 & & & \\
\hline & Model-1 & Lin-TD-CV & 0.881 & 0.400 & 0.445 & 2.201 & 0.052 & 0.770 & 0.592 & 0.857 \\
\hline & & Lin-TD-ADC & 20.204 & 6.783 & 0.602 & 2.979 & $0.014^{*}$ & & & \\
\hline & & Constant & 8.232 & 4.911 & & 1.676 & 0.125 & & & \\
\hline (average) & Model-2 & Lin-V-CV & 0.826 & 0.373 & 0.416 & 2.216 & 0.051 & 0.807 & 0.651 & 0.793 \\
\hline$(\mathrm{km} / \mathrm{h})$ & & Lin-V-ADC & 23.880 & 6.920 & 0.648 & 3.451 & $0.006^{*}$ & & & \\
\hline & & Constant & 8.200 & 5.313 & & 1.543 & 0.154 & & & \\
\hline & Model-3 & Non-2-CV & 0.884 & 0.399 & 0.448 & 2.214 & 0.051 & 0.769 & 0.592 & 0.857 \\
\hline & & Non-2-ADC & 20.494 & 6.934 & 0.598 & 2.956 & $0.014^{*}$ & & & \\
\hline & & Constant & 10.150 & 5.918 & & 1.715 & 0.117 & & & \\
\hline & Model-1 & Lin-TD-CV & 0.817 & 0.446 & 0.372 & 1.832 & 0.097 & 0.767 & 0.588 & 0.954 \\
\hline & & Lin-TD-ADC & 24.112 & 7.551 & 0.649 & 3.193 & $0.010^{*}$ & & & \\
\hline & & Constant & 9.934 & 5.879 & & 1.690 & 0.122 & & & \\
\hline $\mathrm{V}_{(\max )}(\mathrm{km} / \mathrm{h})$ & Model-2 & Lin-V-CV & 0.801 & 0.446 & 0.365 & 1.796 & 0.103 & 0.770 & 0.593 & 0.949 \\
\hline & & Lin-V-ADC & 26.126 & 8.283 & 0.640 & 3.154 & $0.010 *$ & & & \\
\hline & & Constant & 10.060 & 5.932 & & 1.696 & 0.121 & & & \\
\hline & Model-3 & Non-2-CV & 0.818 & 0.446 & 0.374 & 1.834 & 0.096 & 0.765 & 0.585 & 0.957 \\
\hline & & Non-2-ADC & 24.464 & 7.741 & 0.645 & 3.160 & $0.010 *$ & & & \\
\hline
\end{tabular}


Table 5 (continued)

\begin{tabular}{|c|c|c|c|c|c|c|c|c|c|c|}
\hline $\begin{array}{l}\text { Dependent } \\
\text { Variable }\end{array}$ & Model & $\begin{array}{l}\text { Predictor } \\
\text { Variables }\end{array}$ & B & $\begin{array}{l}\text { Standard } \\
\text { Error }\end{array}$ & $\beta$ & $\mathbf{t}$ & $\mathbf{p}$ & $\mathbf{R}$ & $\mathbf{R}^{2}$ & $\begin{array}{l}\text { Standard } \\
\text { Error of } \\
\text { Estimate }\end{array}$ \\
\hline \multirow{9}{*}{$\begin{array}{l}\text { Min. } \\
\text { power } \\
\text { (watative) } \\
\text { (watt/kg) }\end{array}$} & \multirow{3}{*}{ Model-1 } & Constant & -5.692 & 5.047 & & -1.128 & 0.286 & \multirow{3}{*}{0.718} & \multirow{3}{*}{0.516} & \multirow{3}{*}{0.813} \\
\hline & & Lin-TD-CV & 0.698 & 0.380 & 0.404 & 1.835 & 0.096 & & & \\
\hline & & Lin-TD-ADC & 16.677 & 6.440 & 0.571 & 2.590 & $0.027^{*}$ & & & \\
\hline & \multirow{3}{*}{ Model-2 } & Constant & -5.662 & 4.335 & & -1.306 & 0.221 & \multirow{3}{*}{0.801} & \multirow{3}{*}{0.642} & \multirow{3}{*}{0.700} \\
\hline & & Lin-V-CV & 0.606 & 0.329 & 0.351 & 1.843 & 0.095 & & & \\
\hline & & Lin-V-ADC & 21.951 & 6.109 & 0.684 & 3.593 & $0.005^{*}$ & & & \\
\hline & \multirow{3}{*}{ Model-3 } & Constant & -5.885 & 5.006 & & -1.176 & 0.267 & \multirow{3}{*}{0.723} & \multirow{3}{*}{0.523} & \multirow{3}{*}{0.808} \\
\hline & & Non-2-CV & 0.706 & 0.376 & 0.411 & 1.877 & 0.090 & & & \\
\hline & & Non-2-ADC & 17.022 & 6.533 & 0.570 & 2.606 & $0.026^{*}$ & & & \\
\hline \multirow{9}{*}{$\begin{array}{l}\text { Max. } \\
\text { power }_{\text {(relative) }} \\
\text { (watt/kg) }\end{array}$} & \multirow{3}{*}{ Model-1 } & Constant & -7.583 & 7.459 & & -1.017 & 0.333 & \multirow{3}{*}{0.750} & \multirow{3}{*}{0.562} & \multirow{3}{*}{1.202} \\
\hline & & Lin-TD-CV & 0.928 & 0.562 & 0.346 & 1.652 & 0.130 & & & \\
\hline & & Lin-TD-ADC & 29.310 & 9.518 & 0.645 & 3.080 & $0.012^{*}$ & & & \\
\hline & \multirow{3}{*}{ Model-2 } & Constant & -7.904 & 7.383 & & -1.071 & 0.310 & \multirow{3}{*}{0.755} & \multirow{3}{*}{0.570} & \multirow{3}{*}{1.192} \\
\hline & & Lin-V-CV & 0.911 & 0.560 & 0.339 & 1.627 & 0.135 & & & \\
\hline & & Lin-V-ADC & 31.895 & 10.403 & 0.639 & 3.066 & $0.012^{*}$ & & & \\
\hline & \multirow{3}{*}{ Model-3 } & Constant & -7.706 & 7.473 & & -1.031 & 0.327 & \multirow{3}{*}{0.748} & \multirow{3}{*}{0.560} & \\
\hline & & Non-2-CV & 0.931 & 0.562 & 0.348 & 1.657 & 0.129 & & & 1.206 \\
\hline & & Non-2-ADC & 29.740 & 9.753 & 0.641 & 3.049 & $0.012^{*}$ & & & \\
\hline & & Constant & -7.291 & 5.811 & & -1.255 & 0.238 & & & \\
\hline & Model-1 & Lin-TD-CV & 0.883 & 0.438 & 0.422 & 2.016 & 0.071 & 0.750 & 0.563 & 0.937 \\
\hline & & Lin-TD-ADC & 21.101 & 7.416 & 0.596 & 2.845 & $0.017^{*}$ & & & \\
\hline Mean & & Constant & -7.413 & 5.362 & & -1.383 & 0.197 & & & \\
\hline power $_{\text {(relative) }}$ & Model-2 & Lin-V-CV & 0.825 & 0.407 & 0.394 & 2.027 & 0.070 & 0.792 & 0.627 & 0.866 \\
\hline (watt/kg) & & Lin-V-ADC & 25.131 & 7.555 & 0.646 & 3.326 & $0.008^{*}$ & & & \\
\hline & & Constant & -7.414 & 5.809 & & -1.276 & 0.231 & & & \\
\hline & Model-3 & Non-2-CV & 0.887 & 0.437 & 0.426 & 2.031 & 0.070 & 0.750 & 0.562 & 0.938 \\
\hline & & Non-2-ADC & 21.396 & 7.581 & 0.592 & 2.822 & $0.018^{*}$ & & & \\
\hline & & Constant & -4.682 & 13.152 & & -0.356 & 0.729 & & & \\
\hline & Model-1 & Lin-TD-CV & 0.392 & 0.991 & 0.100 & 0.396 & 0.701 & 0.602 & 0.362 & 2.121 \\
\hline & & Lin-TD-ADC & 38.975 & 16.783 & 0.588 & 2.322 & $0.043^{*}$ & & & \\
\hline Fatigue & & Constant & -6.274 & 13.978 & & -0.449 & 0.663 & & & \\
\hline index (watt/ & Model-2 & Lin-V-CV & 0.574 & 1.061 & 0.146 & 0.542 & 0.600 & 0.527 & 0.277 & 2.257 \\
\hline $\mathrm{sec})$ & & Lin-V-ADC & 35.738 & 19.695 & 0.491 & 1.815 & 0.100 & & & \\
\hline & & Constant & -4.883 & 13.100 & & -0.373 & 0.717 & & & \\
\hline & Model-3 & Non-2-CV & 0.392 & 0.985 & 0.101 & 0.398 & 0.699 & 0.605 & 0.366 & 2.115 \\
\hline & & Non-2-ADC & 39.983 & 17.096 & 0.590 & 2.339 & $0.041 *$ & & & \\
\hline
\end{tabular}

Note. ${ }^{*} p<0.05$

aerobic exercise performance [37]. It was reported that $\mathrm{CV}$ was significantly correlated with both maximal lactate steady state and onset of blood lactate [38]. Also, performance at $3000 \mathrm{~m}$ running was closely related to $\mathrm{CV}$ parameter [39]. These findings support that $\mathrm{CV}$ is an aerobic performance indicator. In our study, it was determined that $\mathrm{CV}$ parameter of three models correlated with maximum velocity at $800 \mathrm{~m}$ test $(\mathrm{p}<0.05)$ (table $3)$. However, it was seen that the significance level of correlation was not high (0.44-0.49 range of $p$ value). In this context, it may be said that the $800 \mathrm{~m}$ test is an anaerobic test dominantly and the contribution on test performance of aerobic fitness is low. Simões et al. [40] found a significant correlation between $\mathrm{CV}$ and $500 \mathrm{~m}$,
3 and $10 \mathrm{~km}$ running velocity and this result showed parallelism to correlation between $\mathrm{CV}$ and maximum velocity at $800 \mathrm{~m}$ test in our study. Particularly, the findings of our study were similar to correlation between $\mathrm{CV}$ and $500 \mathrm{~m}$ running velocity in mentioned study. This relationship in mentioned study is remarkable although the anaerobic contribution to $500 \mathrm{~m}$ performance is higher than $800 \mathrm{~m}$ test. Similarly, Bosquet et al. [41] reported that the $40-62 \%$ of variance in velocity at $800 \mathrm{~m}$ running was explained by $\mathrm{CV}$ estimates of five mathematical models. The CV and ADC estimates of three models used in our study predicted significantly maximum velocity at 800 $\mathrm{m}$ test and both parameters explained $73-76.8 \%$ of total variance in mentioned variable (table 6). It was seen that 
Table 6. The Regression Analysis of Effect on $800 \mathrm{~m}$ Test Parameters of CV and ADC as Predictor Variables

\begin{tabular}{|c|c|c|c|c|c|c|c|c|c|c|}
\hline $\begin{array}{l}\text { Dependent } \\
\text { Variable }\end{array}$ & Model & $\begin{array}{l}\text { Predictor } \\
\text { Variables }\end{array}$ & B & $\begin{array}{l}\text { Standart } \\
\text { Error }\end{array}$ & $\beta$ & $\mathbf{t}$ & $p$ & $\mathbf{R}$ & $\mathbf{R}^{2}$ & $\begin{array}{l}\text { Standard } \\
\text { Error of } \\
\text { Estimate }\end{array}$ \\
\hline \multirow{9}{*}{$t(\mathrm{sec})$} & \multirow{3}{*}{ Model-1 } & Constant & 277.148 & 31.199 & & 8.883 & $0.000 *$ & \multirow{3}{*}{0.871} & \multirow{3}{*}{0.759} & \multirow{3}{*}{5.032} \\
\hline & & Lin-TD-CV & -6.160 & 2.351 & -0.408 & -2.621 & $0.026^{*}$ & & & \\
\hline & & Lin-TD-ADC & -190.976 & 39.813 & -0.746 & -4.797 & $0.001 *$ & & & \\
\hline & \multirow{3}{*}{ Model-2 } & Constant & 278.619 & 30.667 & & 9.085 & $0.000 *$ & \multirow{3}{*}{0.875} & \multirow{3}{*}{0.766} & \multirow{3}{*}{4.953} \\
\hline & & Lin-V-CV & -6.000 & 2.327 & -0.396 & -2.579 & $0.027^{*}$ & & & \\
\hline & & Lin-V-ADC & -207.828 & 43.209 & -0.739 & -4.810 & $0.001^{*}$ & & & \\
\hline & \multirow{3}{*}{ Model-3 } & Constant & 276.729 & 32.118 & & 8.616 & $0.000 *$ & \multirow{3}{*}{0.862} & \multirow{3}{*}{0.744} & \multirow{3}{*}{5.186} \\
\hline & & Non-2-CV & -6.101 & 2.414 & -0.405 & -2.527 & $0.030 *$ & & & \\
\hline & & Non-2-ADC & -192.589 & 41.915 & -0.737 & -4.595 & $0.001 *$ & & & \\
\hline \multirow{9}{*}{$\begin{array}{l}\mathrm{V}_{\text {(average) }} \\
(\mathrm{km} / \mathrm{h})\end{array}$} & \multirow{3}{*}{ Model-1 } & Constant & 16.620 & 8.354 & & 1.990 & 0.075 & \multirow{3}{*}{0.519} & \multirow{3}{*}{0.269} & \multirow{3}{*}{1.347} \\
\hline & & Lin-TD-CV & -0.218 & 0.629 & -0.094 & -0.347 & 0.736 & & & \\
\hline & & Lin-TD-ADC & 20.290 & 10.660 & 0.516 & 1.903 & 0.086 & & & \\
\hline & \multirow{3}{*}{ Model-2 } & Constant & 17.214 & 8.363 & & 2.058 & 0.067 & \multirow{3}{*}{0.515} & \multirow{3}{*}{0.265} & \multirow{3}{*}{1.350} \\
\hline & & Lin-V-CV & -0.293 & 0.635 & -0.126 & -0.462 & 0.654 & & & \\
\hline & & Lin-V-ADC & 22.165 & 11.783 & 0.513 & 1.881 & 0.089 & & & \\
\hline & \multirow{3}{*}{ Model-3 } & Constant & 16.997 & 8.426 & & 2.017 & 0.071 & \multirow{3}{*}{0.504} & \multirow{3}{*}{0.254} & \multirow{3}{*}{1.360} \\
\hline & & Non-2-CV & -0.242 & 0.633 & -0.105 & -0.383 & 0.710 & & & \\
\hline & & Non-2-ADC & 20.093 & 10.996 & 0.500 & 1.827 & 0.098 & & & \\
\hline \multirow{9}{*}{$\begin{array}{l}V_{(\max )} \\
(\mathrm{km} / \mathrm{h})\end{array}$} & \multirow{3}{*}{ Model-1 } & Constant & -9.908 & 8.228 & & -1.204 & 0.256 & \multirow{3}{*}{0.854} & \multirow{3}{*}{0.730} & \\
\hline & & Lin-TD-CV & 1.947 & 0.620 & 0.517 & 3.141 & $0.011^{*}$ & & & 1.327 \\
\hline & & Lin-TD-ADC & 41.474 & 10.500 & 0.650 & 3.950 & $0.003^{*}$ & & & \\
\hline & & Constant & -10.023 & 7.615 & & -1.316 & 0.217 & & & \\
\hline & Model-2 & Lin-V-CV & 1.861 & 0.578 & 0.493 & 3.221 & $0.009 *$ & 0.876 & 0.768 & 1.229 \\
\hline & & Lin-V-ADC & 47.182 & 10.729 & 0.674 & 4.398 & $0.001 *$ & & & \\
\hline & & Constant & -10.215 & 8.060 & & -1.267 & 0.234 & & & \\
\hline & Model-3 & Non-2-CV & 1.950 & 0.606 & 0.520 & 3.219 & $0.009 *$ & 0.860 & 0.740 & 1.301 \\
\hline & & Non-2-ADC & 42.619 & 10.519 & 0.654 & 4.052 & $0.002 *$ & & & \\
\hline
\end{tabular}

Note. ${ }^{*} \mathrm{p}<0.05$

the finding of our study was similar to results of Bosquet et al. [41].

It was found that the time and distance values of test performed with $120 \%$ of maximal oxygen uptake velocity were correlated with curvature constant (W' parameter corresponded to ADC) of Lin-TD and Lin-V models in professional young soccer players [42]. The ADC parameter was a significant predictor of the most parameters of RAST (table 5) and $800 \mathrm{~m}$ tests (table 6) in our study and this finding was in agreement with results of mentioned study. These findings show that the effect of $\mathrm{ADC}$ is too distinct in anaerobic exercises. Beck et al. [43] found high and significant correlation $(r=0.68$ 0.83 range, $p<0.05$ ) between RAST power and maximum velocity parameters and times of short distance anaerobic running (50, m running) [43]. Also, the related study reported a significant correlation between mean power at RAST and time of $300 \mathrm{~m}$ running. However, it was determined that the correlation between ADC (constant curvature (W') in related study) and times of 50, 100 and $300 \mathrm{~m}$ runnings was not significant in mentioned study. It was found a significant relationship between $800 \mathrm{~m}$ performance and ADC in our study (table 3 ). The discrepancy of findings may be arisen from difference of running distances $(800 \mathrm{~m} \mathrm{v} 50,100$ and $300 \mathrm{~m}$ ) in these studies. It may be indicated that $800 \mathrm{~m}$ performance may be highly affected by ADC.

Chatzakıs et al. [44] reported that there was a significant correlation between RAST minimum and mean power parameters and 300 and $1000 \mathrm{~m}$ running time in children and young adolescents. In mentioned study, it was reported that maximum power parameters of RAST were only correlated with $300 \mathrm{~m}$ running time. It was found that RAST parameters were significant predictors of time and maximum velocity at $800 \mathrm{~m}$ test in our study (table 7). The $800 \mathrm{~m}$ running test is dominantly anaerobic test. The parameters of RAST involving repeated explosive sprints are indicators of anaerobic exercises such as 800 and $1000 \mathrm{~m}$ running. However, maximum power parameter of RAST is the highest power in exercise. Therefore, it may be said that maximum power parameter of RAST may be more dominant in exercises requiring high contribution of anaerobic energy system such as $300 \mathrm{~m}$ running. Zagatto et al. [45] researched relationship RAST parameters and results of Hoff test (a soccer-specific test developed by Hoff et al. [46] for anaerobic fitness level in soccer players) in professional soccer players and found no significant correlation between test results. It was 
Table 7. The Regression Analysis of Effect on $800 \mathrm{~m}$ Test Parameters of RAST Parameters as Predictor Variables

\begin{tabular}{|c|c|c|c|c|c|c|c|c|c|}
\hline $\begin{array}{l}\text { Dependent } \\
\text { Variable }\end{array}$ & Model & $\begin{array}{l}\text { Predictor } \\
\text { Variables }\end{array}$ & B & $\begin{array}{l}\text { Standard } \\
\text { Error }\end{array}$ & $\beta$ & $t$ & p & $\mathbf{R}^{2}$ & $\begin{array}{l}\text { Standard } \\
\text { Error of } \\
\text { Estimate }\end{array}$ \\
\hline \multirow{16}{*}{$\mathrm{t}(\mathrm{sec})$} & \multirow{2}{*}{ Model-1 } & Constant & 10.394 & 32.047 & & 0.324 & 0.752 & \multirow{2}{*}{0.650} & \multirow{2}{*}{5.781} \\
\hline & & t(average) & 27.613 & 6.112 & 0.806 & 4.518 & $0.001^{*}$ & & \\
\hline & \multirow{2}{*}{ Model-2 } & Constant & 10.753 & 31.875 & & 0.337 & 0.742 & \multirow{2}{*}{0.651} & \multirow{2}{*}{5.770} \\
\hline & & $\mathrm{t}($ total $)$ & 4.593 & 1.014 & 0.807 & 4.531 & $0.001 *$ & & \\
\hline & \multirow{2}{*}{ Model-3 } & Constant & 302.458 & 33.557 & & 9.013 & $0.000^{*}$ & \multirow{2}{*}{0.638} & \multirow{2}{*}{5.881} \\
\hline & & $\mathrm{V}_{\text {(average) }}$ & -6.093 & 1.385 & -0.799 & -4.400 & $0.001 *$ & & \\
\hline & \multirow{2}{*}{ Model-4 } & Constant & 301.431 & 31.417 & & 9.594 & $0.000^{*}$ & \multirow{2}{*}{0.664} & \multirow{2}{*}{5.659} \\
\hline & & $\mathrm{V}(\max )$ & -5.615 & 1.203 & -0.815 & -4.667 & $0.001^{*}$ & & \\
\hline & \multirow{2}{*}{ Model-5 } & Constant & 198.001 & 13.460 & & 14.711 & $0.000^{*}$ & \multirow{2}{*}{0.487} & \multirow{2}{*}{7.000} \\
\hline & & Min. power ${ }_{\text {(relative) }}$ & -6.108 & 1.892 & -0.698 & -3.229 & $0.008^{*}$ & & \\
\hline & \multirow{2}{*}{ Model-6 } & Constant & 204.570 & 11.171 & & 18.312 & $0.000^{*}$ & \multirow{2}{*}{0.646} & \multirow{2}{*}{5.809} \\
\hline & & Max. power ${ }_{\text {(relative) }}$ & -4.530 & 1.010 & -0.804 & -4.484 & $0.001^{*}$ & & \\
\hline & \multirow{2}{*}{ Model-7 } & Constant & 205.360 & 11.902 & & 17.254 & $0.000^{*}$ & & \\
\hline & & Mean power ${ }_{\text {(relative) }}$ & -5.709 & 1.336 & -0.790 & -4.273 & $0.001 *$ & 0.624 & 5.990 \\
\hline & Model-8 & Constant & 178.091 & 8.165 & & 21.811 & $0.000^{*}$ & 0.437 & 7332 \\
\hline & iviouer-o & Fatigue index & -2.549 & 0.873 & -0.661 & -2.920 & $0.014^{*}$ & 0.451 & 1.352 \\
\hline & Model-1 & Constant & 27.722 & 7.828 & & 3.542 & $0.005^{*}$ & 0116 & 1412 \\
\hline & IVIoder-1 & $\mathrm{t}$ (average) & -1.798 & 1.493 & -0.341 & -1.204 & 0.254 & 0.110 & 1.412 \\
\hline & Model-2 & Constant & 27.710 & 7.798 & & 3.553 & $0.005^{*}$ & 0 117 & 1411 \\
\hline & IVIoder-Z & $t($ total $)$ & -0.299 & 0.248 & -0.342 & -1.207 & 0.253 & $0.11 /$ & 1.411 \\
\hline & & Constant & 8.790 & 8.076 & & 1.088 & 0.300 & & \\
\hline & IVlodel-3 & $\mathrm{V}_{\text {(average) }}$ & 0.393 & 0.333 & 0.335 & 1.180 & 0.263 & 0.112 & 1.415 \\
\hline & Model-4 & Constant & 6.220 & 7.499 & & 0.829 & 0.425 & 0191 & 1350 \\
\hline & IVIodel-4 & $V(\max )$ & 0.464 & 0.287 & 0.438 & 1.614 & 0.135 & 0.191 & 1.350 \\
\hline$(\mathrm{km} / \mathrm{h})$ & Model-5 & Constant & 16.439 & 2.832 & & 5.805 & $0.000^{*}$ & 0.039 & 1472 \\
\hline & IVIouer-כ & Min. power (relative) & 0.265 & 0.398 & 0.197 & 0.667 & 0.519 & 0.039 & $1.4 / 2$ \\
\hline & & Constant & 14.195 & 2.603 & & 5.453 & $0.000^{*}$ & & \\
\hline & IVIodel-b & Max. power ${ }_{\text {(relative) }}$ & 0.376 & 0.235 & 0.434 & 1.597 & 0.139 & 0.188 & 1.353 \\
\hline & Model-7 & Constant & 15.074 & 2.818 & & 5.349 & $0.000^{*}$ & 0109 & 1418 \\
\hline & IVIodel-I & Mean power ${ }_{\text {(relative) }}$ & 0.367 & 0.316 & 0.330 & 1.159 & 0.271 & 0.109 & 1.418 \\
\hline & Model-8 & Constant & 16.049 & 1.518 & & 10.573 & $0.000^{*}$ & 0.177 & 1363 \\
\hline & IVIodel-ব & Fatigue index & 0.249 & 0.162 & 0.420 & 1.537 & 0.153 & $0.1 / 7$ & 1.363 \\
\hline & Model-1 & Constant & 57.869 & 8.979 & & 6.445 & $0.000^{*}$ & 0557 & \\
\hline & IVIodel-1 & $\mathrm{t}$ (average) & -6.372 & 1.712 & -0.746 & -3.721 & $0.003 *$ & 0.551 & 1.619 \\
\hline & Model-2 & Constant & 57.696 & 8.967 & & 6.434 & $0.000^{*}$ & 0.555 & 1623 \\
\hline & & $\mathrm{t}$ (total) & -1.057 & 0.285 & -0.745 & -3.707 & $0.003^{*}$ & בככנט & $1.0<3$ \\
\hline & Model-3 & Constant & -8.873 & 9.564 & & -0.928 & 0.373 & 0.526 & 1676 \\
\hline & IVIoder-s & $\mathrm{V}_{\text {(average) }}$ & 1.379 & 0.395 & 0.725 & 3.494 & $0.005^{*}$ & $0.3<0$ & $1.0 \%$ \\
\hline & Model-4 & Constant & -6.765 & 9.672 & & -0.699 & 0.499 & 0488 & 1742 \\
\hline & IVIodel-4 & $V(\max )$ & 1.199 & 0.370 & 0.698 & 3.237 & $0.008^{*}$ & 0.488 & 1.142 \\
\hline$(\mathrm{km} / \mathrm{h})$ & Model-5 & Constant & 12.801 & 3.033 & & 4.220 & $0.001^{*}$ & 0.580 & 1577 \\
\hline & IVlodel-5 & Min. power $r_{\text {(relative) }}$ & 1.662 & 0.426 & 0.762 & 3.898 & $0.002 *$ & 0.580 & $1.5 / 1$ \\
\hline & & Constant & 14.077 & 3.439 & & 4.093 & $0.002^{*}$ & 0.460 & 1788 \\
\hline & IVIodel-6 & Max. power ${ }_{\text {(relative) }}$ & 0.952 & 0.311 & 0.678 & 3.063 & $0.011^{*}$ & 0.460 & 1.188 \\
\hline & Model-7 & Constant & 13.304 & 3.431 & & 3.877 & $0.003^{*}$ & 0.497 & 1727 \\
\hline & IVIoder-7 & Mean power (relative) & 1.269 & 0.385 & 0.705 & 3.295 & $0.007^{*}$ & 0.491 & 1.121 \\
\hline & Model-8 & Constant & 21.130 & 2.500 & & 8.453 & $0.000^{*}$ & 0.150 & 2.244 \\
\hline & IVIodel-8 & Fatigue index & 0.372 & 0.267 & 0.387 & 1.392 & 0.191 & 0.150 & 2.244 \\
\hline
\end{tabular}

Note. ${ }^{*} p<0.05$ 
indicated that the Hoff test was used for measurement of aerobic fitness level with soccer specific exercises (dribbling and activities with ball) [46]. The RAST power parameters were not significantly correlated with $\mathrm{CV}$ in our study (table 2) and this finding sustained the results of Zagatto et al. [45].

It was indicated that $20 \mathrm{~m}$ sprint time was a powerful predictor of total time and sprint decrement score at RAST test in national level soccer players [47]. Similarly, the ADC parameter was a significant predictor of RAST parameters in our study (table 5). The sprint time decrement index (score developed by Glaister et al. [48]) of RAST had significant correlation with maximum oxygen uptake $\left(\mathrm{VO}_{2} \max \right)$ in low and high level $\mathrm{VO}_{2} \max$ groups (positive correlation for low level $\mathrm{VO}_{2} \max$ group, negative correlation for high level $\mathrm{VO}_{2} \max$ group) but no significant correlation was found for medium level $\mathrm{VO}_{2}$ max group [49]. There was no correlation between $\mathrm{CV}$ and fatigue index of RAST in our study (table 2) and this finding drew parallelism with the correlation result of medium level $\mathrm{VO}_{2}$ max group in mentioned study. The decrement index used in study of Alizadeh et al. [49] was different from fatigue index in our study and this difference might cause discrepancy in results of two studies. Keir et al. [50] indicated that contribution of aerobic metabolism in RAST was higher than Wingate test although there was no significant difference between $\mathrm{VO}_{2}$ max values of two tests. There was no comparison between tests in our study and it was not seen a significant correlation between CV and RAST parameters (table 2). The mentioned study has focused on comparison of RAST and Wingate tests and interpreted aerobic metabolism effects on tests by $\mathrm{VO}_{2}$ max graphs. Our study was based on prediction of RAST parameters by $\mathrm{CV}$ and ADC and it was seen that the CV predicted significantly time parameters $\left(\mathrm{t}_{\text {(average) }}\right.$ and $t_{\text {(total) }}$ ) of RAST (table 5). The only CV effect on time parameters of RAST has sustained findings of Keir et al. [50] emphasizing aerobic contribution in RAST.

In study performed on professional soccer players, it was found that the correlation between RAST parameters and soccer match performance (total distance, maximum speed, high intensity and sprint count during soccer match) was not significant statistically [51]. Although the parameters determined in mentioned study were anaerobic activities except for total distance, the relationship between RAST and match parameters was not found by Redkva et al. [51]. Loures et al. [52] reported that the anaerobic work capacity (equivalent of ADC) of soccer players under seventeen age did not correlate with power and fatigue index parameters of RAST but velocity parameters (mean and maximum velocity) had significant correlation with ADC. Similarly, it was determined a non-significant correlation between RAST and anaerobic running capacity (equivalent of ADC) in male futsal players [53]. Unlike finding of mentioned study, there was a significant correlation between all the RAST parameters and ADC (as an anaerobic parameter) in our study (table 2).

\section{Conclusion}

The CV and ADC parameters are yielded by linear and non-linear mathematical models. The $\mathrm{CV}$ is defined as aerobic fitness indices although ADC is an indicator of distance covered with anaerobic energy sources. The repeated sprint and sprint endurance ability is critical for performance in soccer involving repeated sprints. Therefore, RAST and $800 \mathrm{~m}$ performance that are indirect indicators of anaerobic performance is tried to predict by $\mathrm{CV}$ and ADC parameters in this study. It was found that ADC was a strong indicator of RAST performance. Also, it was seen that $800 \mathrm{~m}$ performance might be predicted by ADC and RAST parameters. CV parameter was not a significant predictor of RAST and $800 \mathrm{~m}$ performance except for time parameters of tests. It may be concluded that ADC may be used as an indicator of repeated sprint and sprint endurance performance while $\mathrm{CV}$ is a determinant of aerobic endurance performance. The CV and ADC parameters may be easily determined by simple methods without expensive measurement equipment and the performance of soccer players may be tracked by these parameters.

\section{Acknowledgements}

This study was written by abridging Erdal Arı, Gökhan Deliceoğlu. No grants or financial aids were taken in this Project.

\section{Financial support}

There is no financial support.

\section{Conflict of interest}

The authors report no conflict of interest. 
1. Walsh ML. Whole body fatigue and critical power. Sports Medicine, 2000; 29(3): 153-166. https://doi.org/10.2165/00007256-200029030-00002

2. Monod H, Scherrer J. The work capacity of a synergic muscular group. Ergonomics, 1965; 8(3): 329-338. https://doi.org/10.1080/00140136508930810

3. Moritani T, Nagata A, Devries HA, Muro M. Critical power as a measure of physical work capacity and anaerobic threshold. Ergonomics, 1981; 24(5): 339-350. https://doi.org/10.1080/00140138108924856

4. Bulbulian R, Wilcox AR, Darabos BL. Anaerobic contribution to distance running performance of trained cross-country athletes. Medicine and Science in Sports and Exercise, 1986; 18(1): 107-113. https://doi.org/10.1249/00005768-198602000-00018

5. Housh DJ, Housh TJ, Bauge SM. The accuracy of the critical power test for predicting time to exhaustion during cycle ergometry. Ergonomics, 1989; 32(8): 997-1004. https://doi.org/10.1080/00140138908966860

6. Bulbulian R, Jeong JW, Murphy M. Comparison of anaerobic components of the Wingate and Critical Power tests in males and females. Medicine and Science in Sports and Exercise, 1996; 28(10): 1336-1341. https://doi.org/10.1097/00005768-199610000-00020

7. Hughson RL, Orok CJ, Staudt LE. A high velocity treadmill running test to assess endurance running potential. International Journal of Sports Medicine, 1984; 5(01): 23-25. https://doi.org/10.1055/s-2008-1025875

8. Hopkins WG, Edmond IM, Hamilton BH, Macfarlane DJ, Ross BH. Relation between power and endurance for treadmill running of short duration. Ergonomics, 1989; 32(12): 15651571. https://doi.org/10.1080/00140138908966925

9. Bull AJ, Housh TJ, Johnson GO, Rana SR. Physiological responses at five estimates of critical velocity. European Journal of Applied Physiology, 2008; 102(6): 711-720. https://doi.org/10.1007/s00421-007-0649-7

10.Fukuda DH, Smith AE, Kendall KL, Dwyer TR, Kerksick CM, Beck TW, Cramer JT, Stout JR. The effects of creatine loading and gender on anaerobic running capacity. The Journal of Strength and Conditioning Research, 2010;24(7): 1826-1833. https://doi.org/10.1519/JSC.0b013e3181e06d0e

11.Hill DW, Ferguson CS. A physiological description of critical velocity. European Journal of Applied Physiology and Occupational Physiology, 1999; 79(3): 290-293. https://doi.org/10.1007/s004210050509

12.Wragg CB, Maxwell NS, Doust JH. Evaluation of the reliability and validity of a soccer-specific field test of repeated sprint ability. European Journal of Applied Physiology, 2000; 83(1): 77-83. https://doi.org/10.1007/s004210000246

13.Rampinini E, Bishop D, Marcora SM, Bravo DF, Sassi $\mathrm{R}$, Impellizzeri FM. Validity of simple field tests as indicators of match-related physical performance in top-level professional soccer players. International Journal of Sports Medicine, 2007; 28(03): 228-235. https://doi.org/10.1055/s-2006-924340

14.Impellizzeri FM, Rampinini E, Castagna C, Bishop D, Bravo DF, Tibaudi A, Wisloff U. Validity of a repeated-sprint test for football. International Journal of Sports Medicine, 2008; 29(11): 899-905. https://doi.org/10.1055/s-2008-1038491

15.Buchheit M, Mendez-Villanueva A, Delhomel G, Brughelli M, Ahmaidi S. Improving repeated sprint ability in young elite soccer players: repeated shuttle sprints vs. explosive strength training. The Journal of Strength and Conditioning Research, 2010; 24(10): 2715-2722. https://doi.org/10.1519/JSC.0b013e3181bf0223

16.Haugen TA, Tønnessen E, Seiler S. Anaerobic performance testing of professional soccer players 1995-2010. International Journal of Sports Physiology and Performance, 2013; 8(2): 148-156. https://doi.org/10.1123/ijspp.8.2.148

17.Penteado R, Salvador AF, Corvino RB, Cruz R, Lisbôa FD, Caputo F, De Lucas RD. Physiological responses at critical running speed during continuous and intermittent exhaustion tests. Science and Sports, 2014; 29(6): 99-105. https://doi.org/10.1016/j.scispo.2014.02.003

18.Draper N, Whyte G. Here's a new running based test of anaerobic performance for which you need only a stopwatch and a calculator. Peak Performance, 1997; 96: 3-5.

19.Zagatto AM, Beck WR, Gobatto CA. Validity of the running anaerobic sprint test for assessing anaerobic power and predicting short-distance performances. The Journal of Strength and Conditioning Research, 2009;23(6): 1820-1827. https://doi.org/10.1519/JSC.0b013e3181b3df32

20.Harman EA. The measurement of human mechanical power. In: Maud PJ, Foster C, editors. Physiological assessment of human fitness. Champaign, IL: Human Kinetics; 1995: 87113.

21.Housh DJ, Housh TJ, Bauge SM. (1990). A methodological consideration for the determination of critical power and anaerobic work capacity. Research Quarterly for Exercise and Sport, 1990; 61(4): 406-409. https://doi.org/10.1080/02701367.1990.10607506

22.Jenkins DG, Quigley BM. Blood lactate in trained cyclists during cycle ergometry at critical power. European Journal of Applied Physiology and Occupational Physiology, 1990; 61(3-4): 278-283. https://doi.org/10.1007/BF00357613

23.Hill DW. The critical power concept. Sports Medicine, 1993; 16(4): 237-254. https://doi.org/10.2165/00007256-199316040-00003

24.Gaesser GA, Carnevale TJ, Garfinkel A, Walter DO, Womack CJ. Estimation of critical power with nonlinear and linear models. Medicine and Science in Sports and Exercise, 1995; 27(10): 1430-1438.

25.Kranenburg KJ, Smith DJ. Comparison of critical speed determined from track running and treadmill tests in elite runners. Medicine and Science in Sports and Exercise, 1996; 28(5): 614-618. https://doi.org/10.1097/00005768-199605000-00013

26.Florence SL, Weir JP. Relationship of critical velocity to marathon running performance. European Journal of Applied Physiology and Occupational Physiology, 1997; 75(3), 274-278. https://doi.org/10.1007/s004210050160

27.Housh TJ, Cramer JT, Bull AJ, Johnson GO, Housh DJ. The effect of mathematical modeling on critical velocity. European Journal of Applied Physiology, 2001; 84(5): 469-475. https://doi.org/10.1007/s004210000375

28. Whipp BJ, Huntsman DJ, Storer TW, Lamarra N, Wasserman $\mathrm{K}$. A constant which determines the duration of tolerance to high-intensity work. Proceedings of Federation of American Societies for Experimental Biology; 1982; 41(5): 1591-1591.

29.Poole DC, Ward SA, Gardner GW, Whipp BJ. Metabolic and respiratory profile of the upper limit for prolonged exercise in man. Ergonomics, 1988; 31(9): 1265-1279. https://doi.org/10.1080/00140138808966766

30.Bull AJ, Housh TJ, Johnson GO, Perry SR. Effect of mathematical modeling on the estimation of critical power. Medicine and Science in Sports and Exercise, 2000; 32(2): 526. 
https://doi.org/10.1097/00005768-200002000-00040

31.Carter H, Pringle JS, Jones AM, Doust JH. Oxygen uptake kinetics during treadmill running across exercise intensity domains. European Journal of Applied Physiology, 2002; 86(4): 347-354. https://doi.org/10.1007/s00421-001-0556-2

32.Gaesser GA, Carnevale TJ, Garfinkel A, Walter DO. Modeling of the power-endurance relationship for highintensity exercise. Abstract. Medicine and Science in Sports and Exercise, 1990; 22(2): 16.

33.Smith JC, Hill DW. Mathematical Models of the PowerTime Relationship in High Intensity Cycling: 443. Abstract. Medicine and Science in Sports and Exercise, 1992; 24(5): 74.

34.Morton RH. A 3-parameter critical power model. Ergonomics, 1996; 39(4): 611-619. https://doi.org/10.1080/00140139608964484

35.Berthoin S, Baquet G, Dupont G, Blondel N, Mucci P. Critical velocity and anaerobic distance capacity in prepubertal children. Canadian Journal of Applied Physiology, 2003; 28(4): 561-575. https://doi.org/10.1139/h03-043

36.Kendall KL, Fukuda DH, Smith AE, Cramer JT, Stout JR. Predicting maximal aerobic capacity (VO2max) from the critical velocity test in female collegiate rowers. Journal of Strength and Conditioning Research, 2012; 26(3): 733-738. https://doi.org/10.1519/JSC.0b013e318225f3ac

37.Gamelin FX, Coquart J, Ferrari N, Vodougnon H, Matran $\mathrm{R}$, Leger L, Bosquet L. Prediction of one-hour running performance using constant duration tests. The Journal of Strength and Conditioning Research, 2006; 20(4): 735-739. https://doi.org/10.1519/r-17905.1

38.Denadai BS, Gomide E, Greco CC. Validity of critical velocity to determine the maximal lactate steady state in soccer players. 5th World Congress on Science and Football; 2003 April 11-15; Lisboa, Portugal.

39.Massini DA, Caritá RA, Da Cruz Siqueira LO, Simionato AR, Denadai BS, Pessôa Filho DM. Assessment of critical velocity in track and treadmill: physiological profiles and relationship with 3000-meter performance. Revista Brasileira de Cineantropometria and Desempenho Humano, 2018; 20(5): 432-444. https://doi.org/10.5007/1980-0037.2018v20n5p432

40.Simões HG, Denadai BS, Baldissera V, Campbell CSG, Hill DW. Relationships and significance of lactate minimum, critical velocity, heart rate deflection and $3000 \mathrm{~m}$ tracktests for running. Journal of Sports Medicine and Physical Fitness, 2005; 45(4): 441.

41.Bosquet L, Duchene A, Lecot F, Dupont G, Leger L. V max estimate from three-parameter critical velocity models: validity and impact on $800 \mathrm{~m}$ running performance prediction. European Journal of Applied Physiology, 2006; 97(1): 34. https://doi.org/10.1007/s00421-006-0143-7

42.Loures JP, Kalva Filho CA, Franco VH, Kaminagakura EI, Zagatto AM, Papoti M. Correlation between the curvature constant parameter (W') from the velocity-exhaustion time relationship, maximal accumulated oxygen deficit and performance in professional soccer players. Journal of Exercise Physiology Online, 2012; 15(5).
43.Beck WR, Zagatto AM, Gobatto CA. (2014). Repeated sprint ability tests and intensity-time curvature constant to predict short-distance running performances. Sport Sciences for Health, 2014; 10(2): 105-110. https://doi.org/10.1007/s11332-014-0180-2

44.Chatzakis P, Zanni E, Paradisis G, Argeitaki P, Zacharogiannis E. Determinants of 300 and 1000 meters running performance in young track and field athletes. Journal of Physical Education, 2019; 6(1): 21-27. https://doi.org/10.15640/jpesm.v6n1a3

45.Zagatto AM, Miyagi WE, Brisola GMP, Milioni F, Da Silva ASR, Santiago PRP, Papoti M. Correlation between Hoff test performance, body composition and aerobic and anaerobic fitness in professional soccer players. Sport Sciences for Health, 2015; 11(1): 73-79. https://doi.org/10.1007/s11332-014-0210-0

46.Hoff J, Wisløff U, Engen LC, Kemi OJ, Helgerud J. Soccer specific aerobic endurance training. British Journal of Sports Medicine, 2012; 36(3): 218-221. https://doi.org/10.1136/bjsm.36.3.218

47.Brocherie F, Girard O, Forchino F, Al Haddad H, Dos Santos GA, Millet GP. Relationships between anthropometric measures and athletic performance, with special reference to repeated-sprint ability, in the Qatar national soccer team. Journal of Sports Sciences, 2014; 32(13): 1243-1254. https://doi.org/10.1080/02640414.2013.862840

48.Glaister M, Stone MH, Stewart AM, Hughes M, Moir GL. The reliability and validity of fatigue measures during shortduration maximal-intensity intermittent cycling. The Journal of Strength and Conditioning Research, 2004; 18(3): 459462.

49.Alizadeh R, Hovanloo F, Safania AM. The relationship between aerobic power and repeated sprint ability in young soccer players with different levels of $\mathrm{VO}_{2}$ Max. Journal of Physical Education and Sport/Citius Altius Fortius, 2010; 27(2).

50.Keir DA, Thériault F, Serresse O. Evaluation of the runningbased anaerobic sprint test as a measure of repeated sprint ability in collegiate-level soccer players. The Journal of Strength and Conditioning Research, 2013;27(6): 1671-1678. https://doi.org/10.1519/JSC.0b013e31827367ba

51.Redkva PE, Paes MR, Fernandez R, Da-Silva SG. Correlation betweenmatchperformanceandfieldtestsinprofessionalsoccer players. Journal of Human Kinetics, 2018; 62(1): 213-219. https://doi.org/10.1515/hukin-2017-0171

52.Loures JP, Kalva Filho CA, Franco VH, Bittencourt DA, Kaminagakura EI, Papoti M. Correlation between running anaerobic sprint test and anaerobic work capacity in soccer players. International Journal of Exercise Science: Conference Proceedings, 2012; 1(1): Article 42.

53. Messias LHD, De Andrade VC, Rosante K, De Lima TB, Santa Cruz RAR, De Oliveira RM, De Barros Manchado-Gobatto F. Running anaerobic sprint test, lactate minimum and critical velocity protocol in shuttle futsal testing. Central European Journal of Sport Sciences and Medicine, 2015; 12: 5-15. https://doi.org/10.18276/cej.2015.4-01 


\section{Information about the authors:}

Erdal Arı; (Corresponding author); https://orcid.org/0000-0003-1348-7930; arierdal@hotmail.com; Physical Education and Sports School, Ordu University; Ordu, Turkey.

Gökhan Deliceoğlu; https://orcid.org/0000-0003-2459-9209; deliceoglugokhan@hotmail.com; Faculty of Sports Sciences, Gazi University; Gazi, Turkey:

Cite this article as:

Arı E, Deliceoglu G. The prediction of repeated sprint and speed endurance performance by parameters of critical velocity models in soccer. Pedagogy of Physical Culture and Sports, 2021;25(2):132-143.

https://doi.org/10.15561/26649837.2021.0208

This is an Open Access article distributed under the terms of the Creative Commons Attribution License, which permits unrestricted use, distribution, and reproduction in any medium, provided the original work is properly cited (http://creativecommons.org/licenses/by/4.0/deed.en).

Received: 02.10 .2020

Accepted: 08.11.2020; Published: 30.04.2021 